\title{
Comparison between 1.5- and 3-T Magnetic Resonance Acquisitions for Direct Targeting Stereotactic Procedures for Deep Brain Stimulation: A Phantom Study
}

\author{
Gaëtan Poulen ${ }^{\text {a-f }}$ Emilie Chan Seng ${ }^{\text {a-f }}$ Nicolas Menjot De Champfleur ${ }^{g}$ \\ Laura Cif $^{a-f}$ Fabienne Cyprien ${ }^{\text {a- } f}$ Jerome Perez ${ }^{\text {b-f }}$ Philippe Coubes ${ }^{a-f}$

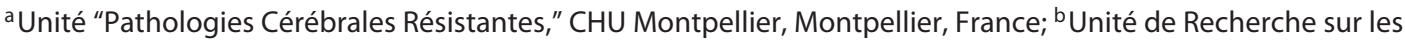

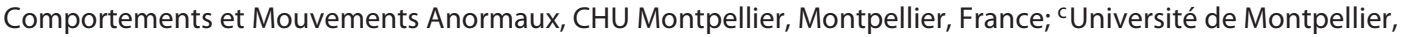 \\ Montpellier, France; ${ }^{d}$ Institut de Génomique Fonctionnelle, Montpellier, France; ${ }^{e}$ CNRS UMR5203, Montpellier, \\ France; ${ }^{f}$ INSERM U661, Montpellier, France; ${ }^{9}$ Unité de Neuroradiologie, CHU Montpellier, Montpellier, France
}

\section{Keywords}

Accuracy $\cdot$ Comparative study $\cdot$ MRI-based study $\cdot$ Phantom study $\cdot$ Stereotactic neurosurgery

\begin{abstract}
Introduction: Deep brain stimulation (DBS) is a well-established treatment for movement disorders. High magnetic fields could have an impact on distortion. We evaluated 1.5and 3-T magnetic resonance imaging (MRI) sequences for accuracy, precision, and trueness of our MRI-guided direct targeting protocol. Methods: Effects of distortion on MR sequences (T1- and T2-weighted sequences) can be evaluated using a dedicated phantom (Elekta). Field strength capabilities were assessed on Siemens Avanto (1.5 T) and Skyra (3 T) scanners. We assessed the precision of our stereotactic MRIguided procedure. Results: We focused on the risk of error due to a high field strength. Error values on the localizer box were between 0.4 and $0.7 \mathrm{~mm}$ at $1.5 \mathrm{~T}$ and between 0.6 and $2 \mathrm{~mm}$ at $3 \mathrm{~T}$. The most accurate 1.5-T sequence is the 3D FLASH T1-weighted sequence, which had an accuracy value of $0.6 \mathrm{~mm}$. At $3 \mathrm{~T}$, the accuracy value of the isotropic 3D FLASH T1-weighted sequence was $1.6 \mathrm{~mm}$. Conclusion: Giv-
\end{abstract}

karger@karger.com

(c) 2020 S. Karger AG, Basel

www.karger.com/sfn

Karger" en the millimetric size of stereotactic targets and electrodes, lead implantation for neuromodulation therapy needs to be accurate. We demonstrate that 3-T imaging could not be used for stereotaxy in our MRI-guided direct targeting protocol because of a risk of error induced by distortion.

ㄷ) 2020 S. Karger AG, Base

\section{Introduction}

Deep brain stimulation (DBS) is a well-established treatment for movement disorders. Its efficacy has been proven in many studies (Globus Pallidus internus [1-4]; subthalamic nucleus [5]; ventral intermediate nucleus of the thalamus [6]). Magnetic resonance imaging (MRI)guided direct targeting under general anesthesia or local anesthesia using microelectrode recording $[7,8]$ and atlas coordinates are used.

Portions of this work were presented in poster form at The MDS 18th International Congress of Parkinson's Disease and Movement Disorders, Stockholm, Sweden, June 9, 2014. 
Fig. 1. Potential sources of errors during MR-guided stereotactic procedures: quality control at every step maximizes precision. a Phantom visualization with the frame. b Verification of the localizer box. c Intraoperative X-ray verification of $y$ and $z$ coordinates (the middle of the lead is in the target in our protocol). $\mathbf{d}$ Checking the geometry of the arc and the straightness of the cannula.

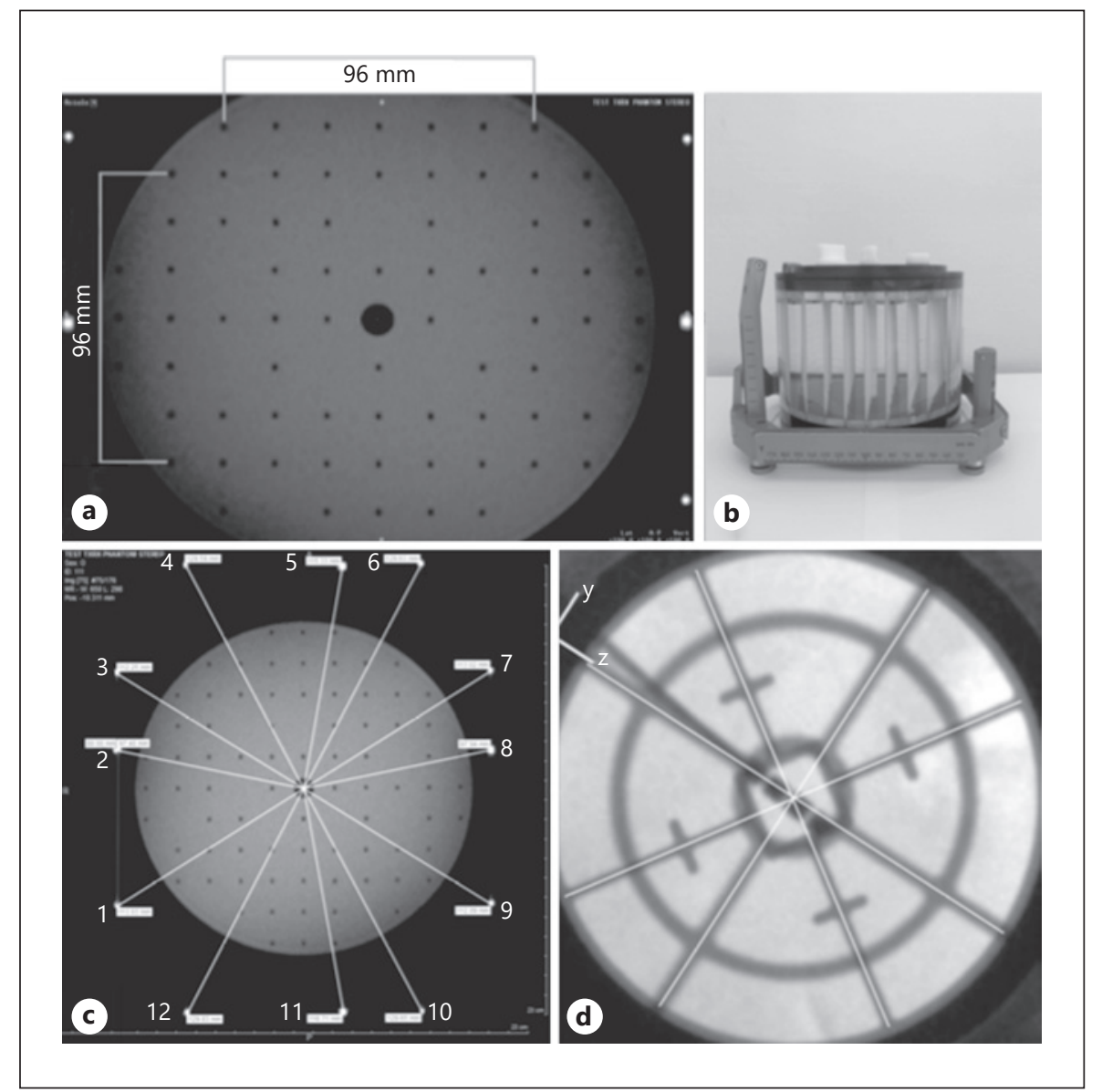

Coubes et al. [9] showed that MRI-guided direct targeting under general anesthesia should be used in stereotaxy, for implanting DBS leads. General anesthesia is more comfortable for the patient (especially in children and hyperkinetic adult patients) and preferable for the surgical team, allowing a better brain volume control and a lower risk of complications [9].

When 3-T MRI became available, an increase in surgical precision was expected due to a better anatomical definition compared to $1.5 \mathrm{~T}$ with benefit in direct targeting surgery. Advantages and pitfalls associated with the use of 3-T scanners have now been well described $[10,11]$. Boutet et al. [12] demonstrated the safety of coils and pulse sequences of 3-T MRI of DBS patients. Voormolen et al. [13] found no visible intracranial distortion in magnetization-prepared T1-weighted 7-T MRI cranial images but considerable extracranial shifts for image-guided cranial neurosurgery.

In a preliminary study, surprisingly, Juhász et al. [14] showed no significant differences in target coordinates comparing 3-T to 1-T magnetic fields for subthalamic nucleus DBS. Our initial experience with 3-T MRI being different from that of others, the potential impact of high magnetic fields on distortion needed to be precisely studied. We have, therefore, evaluated 1.5- and 3-T MR sequences for accuracy, precision, and trueness in our MRIguided direct targeting protocol $[9-11,15]$.

\section{Materials and Methods}

The term "accuracy" used in this article is based on the international ISO-5725 standard definition. Precision is defined by the notion of repeatability (standard deviation [SD]) [16]. The trueness expresses the distance between the statistical mean value and a reference value. Ultimately, accuracy consists of both precision and trueness.

\section{Quantitative MR Phantom Study}

A MR-dedicated cylindrical phantom (Elekta ${ }^{\circledR} \mathrm{AB}$, Stockholm, Sweden; Fig. 1a, b) is centered within the stereotactic Leksell ${ }^{\circledR} \mathrm{G}-$ frame (Elekta ${ }^{\circledR} \mathrm{AB}$ ). This phantom simulates the head of the patient. Filled with water, it contains a set of MRI-compatible tubes aligned in parallel. Tubes are solid and visualized as black hypointense landmarks on axial MR images (T1- and T2-weighted se- 
Table 1. Design of MR sequences studied

\begin{tabular}{|c|c|c|c|c|c|c|c|c|}
\hline $\begin{array}{l}\text { Field } \\
\text { strength }\end{array}$ & $\begin{array}{l}\text { Parameters, } \\
\text { sequence type }\end{array}$ & TA & $\mathrm{FOV}, \mathrm{mm}$ & $\begin{array}{l}\text { Voxel size, } \\
\mathrm{mm}\end{array}$ & $\begin{array}{l}\mathrm{TR}, \\
\mathrm{ms}\end{array}$ & $\begin{array}{l}\mathrm{TE} \\
\mathrm{ms}\end{array}$ & $\begin{array}{l}\text { band- } \\
\text { width/ } \\
\text { pixel, } \mathrm{Hz}\end{array}$ & $\begin{array}{l}\text { Turbo } \\
\text { factor }\end{array}$ \\
\hline \multirow[t]{5}{*}{$1.5 \mathrm{~T}$} & T1-fl3D & $15 \mathrm{mn} 5 \mathrm{~s}$ & $256 \times 256 \times 264$ & $1 \times 1 \times 1.5$ & 17 & 4.76 & 140 & \multirow{5}{*}{10} \\
\hline & T2-TSE & $14 \mathrm{mn} 42 \mathrm{~s}$ & $256 \times 256 \times 45$ & $1 \times 1 \times 1.5$ & 4,200 & 109 & 106 & \\
\hline & T1-MPR iso & $18 \mathrm{mn} 22 \mathrm{~s}$ & $256 \times 256 \times 209$ & $1 \times 1 \times 1 \times 1$ & 2,150 & 8.6 & 170 & \\
\hline & T1-MPR & $9 \mathrm{mn} 12 \mathrm{~s}$ & $256 \times 256 \times 264$ & $1 \times 1 \times 1 \times 1.5$ & 2,150 & 4.2 & 130 & \\
\hline & SWI & $25 \mathrm{mn} 7 \mathrm{~s}$ & $256 \times 256 \times 180$ & $1 \times 1 \times 1.5$ & 49 & 40 & 80 & \\
\hline \multirow[t]{2}{*}{$3 \mathrm{~T}$} & T1-fl3D iso & $10 \mathrm{mn} 30 \mathrm{~s}$ & $256 \times 256 \times 264$ & $0.5 \times 0.5 \times 0.5$ & 11 & 4.92 & 210 & \\
\hline & T1-MPR & $14 \mathrm{mn} 4 \mathrm{~s}$ & $256 \times 256 \times 264$ & $1 \times 1 \times 1.5$ & 2,200 & 2.42 & 250 & \\
\hline
\end{tabular}

Only the first 2 sequences (bold) are routinely used during our stereotactic procedure. TA, time of acquisition; mn, minutes; s, seconds; FOV, field of view; TR, time of repetition; TE, time of echo; TSE, turbo spin echo; iso, isotropic; SWI, susceptibility-weighted scan.

quences). Landmarks are horizontally and vertically equidistant, $16 \mathrm{~mm}$ apart, on axial slices and allow the spatial distribution of error to be determined throughout the image.

MR Image Acquisitions with the Phantom

MR acquisitions were performed with the phantom on a 1.5-T MR scanner (MAGNETOM Avanto ${ }^{\circledR}$ and 3T-MAGNETOM Sky$\mathrm{ra}^{\circledR}$, Siemens Medical Solutions, Erlangen, Germany). The imaging protocol consists of axial T1-weighted scans (T1-fl3D, T1MPRAGE), axial T2-weighted scan (T2-turbo spin echo [TSE]), and axial susceptibility-weighted scan (SWI). Sequences are described in Table 1.

\section{Data Processing}

Six hundred and thirty-two measures were performed on each studied sequence (79 landmarks, 4 planes, 2 coordinates). With magnification, the simplicity of dot positioning in the center of the round surface of each landmark image of the phantom must be pointed out. The very high number of observations allows a powerful statistical analysis.

We simply use the geometric error $e=\sqrt{ } d x^{2}+d y^{2}$, where $d x$ and $d y$ are the absolute difference between measured coordinates and theoretic coordinates of landmarks along the $x$-axis and the $y$-axis, respectively [17-19]. Measurements are performed at the maximum available magnification $(\times 8)$ on the planning station (Framelink software, Medtronic ${ }^{\circledR}$, Minneapolis, USA).

The accuracy value is characterized by the limit of the repeatability threshold. We name this threshold "ê" in this study.

Statistical analysis was performed by Student $t$ test.

\section{Quality of the Stereotactic Space}

The MR localizer box was used to determinate stereotactic coordinates. The software detected the peripheral rods and created a metric system defining the stereotactic space. The N-shaped marker channels allowed the $z$-value to be calculated using markers No. $2,5,8$, and 11 on every MR image. Simultaneously, the other 8 landmarks gave the $x$ - and $y$-coordinates (Fig. 1c).

The center of the phantom was the reference point. The distance between this central point and each peripheral landmark was measured (Fig. 1c) and the absolute error was then calculated. Each side of the localizer box consists of panels (anterior, posterior, left, and right). The mean error of the 4 panels was named "localizer box error." "Panel error" was the mean error of the 3 landmarks on the same panel, and "fiducial error" was the error of each individual landmark.

\section{Surgical Procedure}

Given the specificity of the MR-based stereotactic procedures, it appeared necessary to describe it again here. The systematic doublechecking of manual coordinate settings was necessary and crucial. A 14-mm burrhole allowed a direct visual control during millimetric corticotomy and avoidance of conflict between the cannula and the burrhole edges including the dura (3-mm cross-incision).

Real-time radioscopic (X-ray, lateral view) control was performed during surgery and constituted a crucial step. The cannula and then the lead positions were recorded. Radioscopic control at a very high magnification provided the straightness and the rigidity of the tool $(<0.5 \mathrm{~mm})$. At the same time, the accuracy of the localization of the target was evaluated in the stereotactic space. When removed, the lead positioning in $z$ was fixed by a radioscopic control (Fig. 1d). Subsequently, the effect of brain shift was also monitored if existing. In case of delayed significant shift, the lead's position may slightly deviate from the initial localization of the cannula which remains the reference. The time between the moment of dural opening and the insertion of the cannula was as short as possible to minimize the risk of cerebrospinal fluid leakage and brain shift. Intraoperative profile X-ray allowed the $y$-axis (anteroposterior lead placement) and $z$-axis (depth of the lead) to be monitored in real time during implantation. Checking the setting of the $x$ coordinate (laterality) was performed in the same way intraoperatively by frontal incidence.

Sources of error during this surgical step can be parallax effects, brain density gradients (varying with diseases) which could modify the trajectory, brain shift, size and time of dura opening.

\section{Control of Final Lead Position}

An intraoperative MRI with the frame was performed immediately to verify the position of leads and was fused with the preop- 

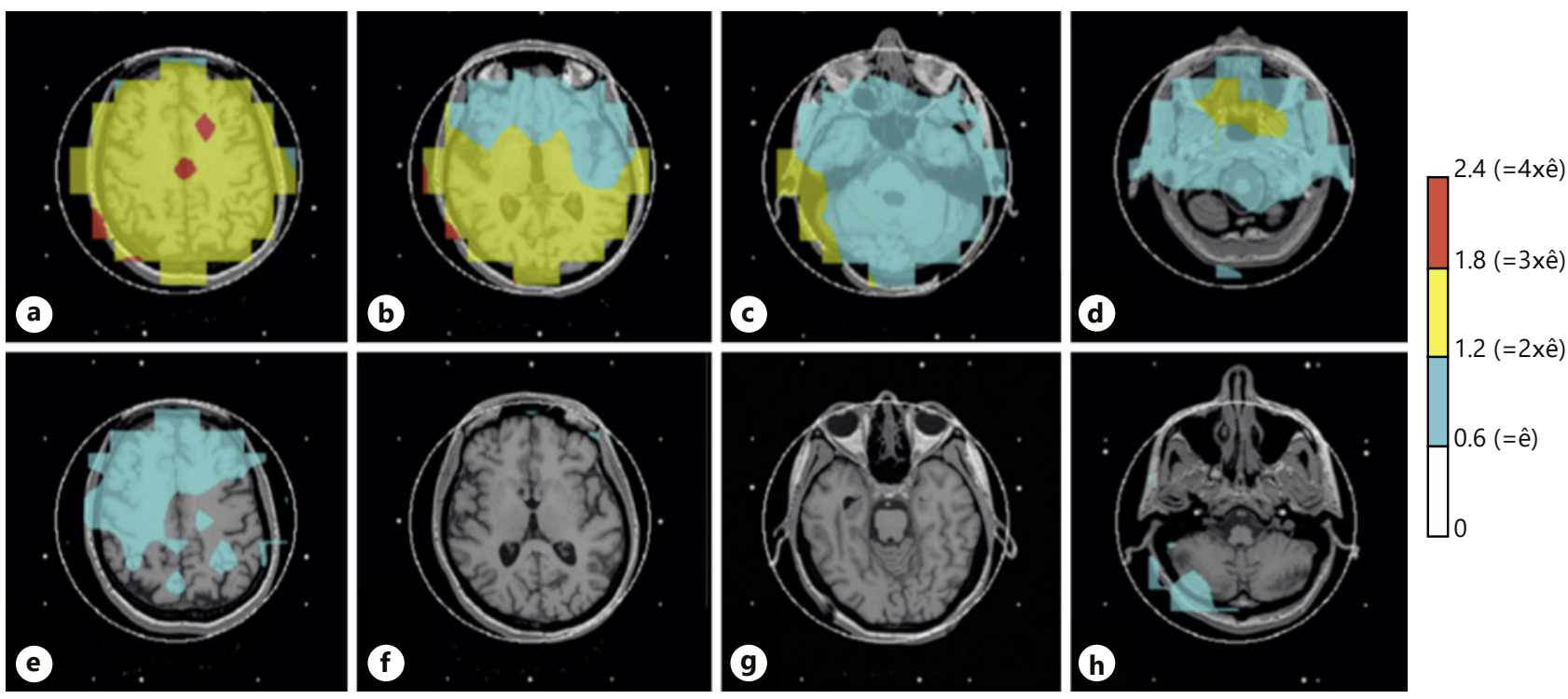

Fig. 2. Superimposed error maps (the white circle shows the phantom location) with T1-weighted FLASH 3D MR images at $3 \mathrm{~T}(\mathbf{a}-\mathbf{d})$ and $1.5 \mathrm{~T}(\mathbf{e}-\mathbf{h})$. Axial distortion maps were generated by linear interpolation between the positional deviation measured at each control point. In-plane error values (significantly superior to the accuracy threshold value of the T1-weighted sequence at $1.5 \mathrm{~T})$ are shown for $z=75 \mathrm{~mm}(\mathbf{a}, \mathbf{e}), z=100 \mathrm{~mm}(\mathbf{f})$, $z=105 \mathrm{~mm}(\mathbf{b}), z=125 \mathrm{~mm}(\mathbf{g}), z=135 \mathrm{~mm}(\mathbf{c}), z=150 \mathrm{~mm}(\mathbf{h})$, and $z=165 \mathrm{~mm}(\mathbf{d})$. There is no significant error for $z=125 \mathrm{~mm}(\mathbf{g})$, corresponding to the ventral part of the grey nuclei.

Table 2. Absolute mean errors of distance between landmarks and the center of the localizer box

\begin{tabular}{lllllll}
\hline Field strength & Sequence type & \multicolumn{2}{l}{$\begin{array}{l}\text { Absolute mean } \\
\text { error } \pm \mathrm{SD}, \mathrm{mm}\end{array}$} & \multicolumn{2}{l}{ Absolute mean error of each panel \pm SD, mm } \\
\cline { 6 - 7 } & & & right & anterior & left & posterior \\
\hline \multirow{2}{*}{$1.5 \mathrm{~T}$} & T1-fl3D & $\mathbf{0 . 6} \pm \mathbf{0 . 3}$ & $\mathbf{0 . 5} \pm \mathbf{0 . 2}$ & $\mathbf{0 . 5} \pm \mathbf{0 . 1}$ & $\mathbf{0 . 3} \pm \mathbf{0 . 1}$ & $\mathbf{1 . 1} \pm \mathbf{0 . 4}$ \\
& T2-TSE & $\mathbf{0 . 4} \pm \mathbf{0 . 1}$ & $\mathbf{0 . 3} \pm \mathbf{0 . 1}$ & $\mathbf{0 . 5} \pm \mathbf{0 . 0 4}$ & $\mathbf{0 . 1} \pm \mathbf{0 . 0 1}$ & $\mathbf{0 . 8} \pm \mathbf{0 . 1}$ \\
& T1-MPR iso & $0.7 \pm 0.3$ & $0.8 \pm 0.2$ & $0.5 \pm 0.1$ & $0.4 \pm 0.1$ & $1.0 \pm 0.5$ \\
& T1-MPR & $0.6 \pm 0.3$ & $0.7 \pm 0.2$ & $0.5 \pm 0.2$ & $0.3 \pm 0.1$ & $1.0 \pm 0.4$ \\
& SWI & $0.7 \pm 0.3$ & $0.6 \pm 0.4$ & $0.9 \pm 0.3$ & $0.4 \pm 0.1$ & $0.8 \pm 0.4$ \\
\hline $3 \mathrm{~T}$ & T1-fl3D iso & $2.0 \pm 1.1$ & $1.8 \pm 0.5$ & $2.8 \pm 1.4$ & $2.3 \pm 0.6$ & $1.2 \pm 0.5$ \\
& T1-MPR & $0.6 \pm 0.3$ & $0.5 \pm 0.1$ & $0.8 \pm 0.4$ & $0.6 \pm 0.2$ & $0.6 \pm 0.3$ \\
\hline
\end{tabular}

Only the first 2 sequences (bold) are routinely used in our stereotactic procedure. Forty-eight measurements were performed for each sequence. SD, standard deviation; TSE, turbo spin echo; iso, isotropic; SWI, susceptibility-weighted scan.

erative MRI. Every time, the automatically calculated error (Framelink software, Medtronic ${ }^{\circledR}$, USA) was above $0.5 \mathrm{~mm}$. Fusion was performed manually.

A final MR control was the only way to exactly localize each electrode in the brain. Particularly, it allowed assessment of the shift (commissures coordinates, target vs. lead's artifact center) and existence of early complications.

\section{Results}

\section{Control of Frame and Localization Box}

Measurements from the localizer box are reported in Table 2. Higher mean "panel error" values were observed on the anterior panel in T1-fl3D (3 T), T1-MPR (3 T), and 
Table 3. The absolute difference between measured and theoretical landmarks' coordinates

\begin{tabular}{lllllll}
\hline $\begin{array}{l}\text { Field } \\
\text { strength }\end{array}$ & $\begin{array}{l}\text { Sequence } \\
\text { type }\end{array}$ & $\begin{array}{l}\text { Mean, } \\
\mathrm{mm}\end{array}$ & $\begin{array}{l}\text { Min., } \\
\mathrm{mm}\end{array}$ & $\begin{array}{l}\text { Max., } \\
\mathrm{mm}\end{array}$ & $\begin{array}{l}\mathrm{SD}, \\
\mathrm{mm}\end{array}$ & $\begin{array}{l}\hat{\mathrm{e}}=\text { mean } \\
+2 \mathrm{SD}, \\
\mathrm{mm}\end{array}$ \\
\hline \multirow{2}{*}{$1.5 \mathrm{~T}$} & T1-fl3D & $\mathbf{0 . 2 5}$ & $\mathbf{0}$ & $\mathbf{0 . 9}$ & $\mathbf{0 . 1 9}$ & $\mathbf{0 . 6}$ \\
& T2-TSE & $\mathbf{0 . 3 5}$ & $\mathbf{0}$ & $\mathbf{0 . 9}$ & $\mathbf{0 . 1 9}$ & $\mathbf{0 . 7}$ \\
& T1-MPR iso & 0.34 & 0 & 1.1 & 0.17 & 0.7 \\
& T1-MPR & 0.29 & 0 & 0.9 & 0.21 & 0.7 \\
& SWI & 0.33 & 0 & 1.4 & 0.24 & 0.8 \\
\hline $3 \mathrm{~T}$ & T1-fl3D iso & 0.7 & 0 & 2.0 & 0.46 & 1.6 \\
& T1-MPR & 0.39 & 0 & 1.5 & 0.28 & 1.0 \\
\hline
\end{tabular}

Only the first 2 sequences (bold) are routinely used in our stereotactic procedure. Six hundred and thirty-two measurements were performed for each sequence. SD, standard deviation; TSE, turbo spin echo; iso, isotropic; SWI, susceptibility-weighted scan.

SWI (1.5 T) sequences. All other sequences showed higher mean "panel error" on the posterior panel, even though the Framelink software only needs left, anterior, and right panels to build the numerical stereotactic space.

The T1-fl3D sequence at $3 \mathrm{~T}$ was the sequence associated with the greatest distortion rate, and the fiducial markers are considerably displaced (absolute mean localizer box error is $2.0 \pm 1.1 \mathrm{~mm}$ ). Regarding our standard surgical procedure, routine T2-TSE $(1.5 \mathrm{~T})$ and T1-fl3D $(1.5 \mathrm{~T})$ sequences displayed a mean localizer box error value of 0.4 and $0.6 \mathrm{~mm}$, respectively. Mean panel error values on each panel used by Framelink (left, anterior, and right) were under $0.5 \mathrm{~mm}$.

\section{Control of MRI}

All sequences were analyzed, and the results are summarized in Table 3. Mean and SD were higher for sequences tested on the 3-T scanner. For 1.5-T measurements, the lowest mean error value was obtained with the FLASH sequence, and the lowest SD value was obtained with the isotropic sequence.

Errors from routine sequences were $0.25 \pm 0.19 \mathrm{~mm}$ (range $0-0.9$ ) and $0.35 \pm 0.19 \mathrm{~mm}$ (range $0-0.9$ ) for T1fl3D and T2-TSE sequences, respectively. Errors from 3 - $\mathrm{T}$ acquisitions were $0.70 \pm 0.46 \mathrm{~mm}$ (range $0-2.0$ ) and $0.39 \pm 0.28 \mathrm{~mm}$ (range $0-1.5$ ) for isotropic T1-fl3D and T1-MPR sequences, respectively. Isotropic sequences were not associated with smaller errors for either field strength.

The reference T1-weighted FLASH sequences at $1.5 \mathrm{~T}$ and $3 \mathrm{~T}$ are compared in Figure 2 by mapping spatial er- rors. Errors were visualized as functions of multiples of the probabilistic ê-threshold (mean +2 SD) from the 1.5$\mathrm{T}$ acquisition. These errors were outside the basal ganglia zone on the 1.5-T sequences, unlike on the 3 - $\mathrm{T}$ sequence where larger errors occurred across the majority of planes (Fig. 2a, b). At 1.5 T, there was no significant error when $z=125 \mathrm{~mm}$ (Fig. 2g), and the highest error values were seen at $z=75 \mathrm{~mm}$ and $z=150 \mathrm{~mm}$ (i.e., at the limits of the volume being studied).

\section{Discussion}

\section{Favoring 1.5-T Magnetic Field}

Outlines of grey nuclei were reported to be easier to define with very high field strength magnets without frame ( $3 \mathrm{~T}$ and $7 \mathrm{~T}$ ) [20-22]. On the contrary, abandoning the stereotactic frame introduces a risk of error that should be carefully evaluated (the impact of head movements on accuracy is unacceptable in stereotaxy for movement disorders).

MR-based targeting methodology does not require the use of atlases. The variability of human brain anatomy is well established as the main pitfall [23] resulting in errors in the actual target location.

Very high field strength scanners have clinical applications in functional neuroimaging, cardiovascular imaging, and MR angiography. Nevertheless, their use is associated with the appearance of specific artifacts caused by an increased signal to noise ratio [24]. Risk of distortion becomes greater and susceptibility effects are amplified. Geometric errors due to distortion caused by the use of very high field strength and the presence of the frame could be reduced, under $1 \mathrm{~mm}$, by using the inverted gradient correction method [25]. With currently available technology, 3-T MRI cannot routinely provide the same level of precision in frame-based stereotaxy as 1.5-T MRI, which is, in plane, $0.6 \mathrm{~mm}$ ( $\mathrm{T} 1-\mathrm{fl} 3 \mathrm{D}$ sequence).

We separately reported errors on the target and on the localizer box. If, in fact, the error of the localizer box (Table 2) was at $0.6 \mathrm{~mm}$ for $3 \mathrm{~T}$, we observed that the mean in-plane error on the target (Table 2) for $3 \mathrm{~T}(0.7 \pm 0.46$ $\mathrm{mm}[0-2.0])$ was much bigger than for $1.5 \mathrm{~T}(0.25 \pm 0.19$ $\mathrm{mm}[0-0.9])$.

Finally, we calculated a maximum deviation of $2 \mathrm{~mm}$ in $3 \mathrm{~T}$ that must be compared to a maximum deviation of $0.9 \mathrm{~mm}$ in $1.5 \mathrm{~T}$. These variable and unpredictable sources of error must be anticipated in the surgical situation: this is why we conclude that if the best accuracy is at 0.6 $\mathrm{mm}, 3-\mathrm{T}$ MRI cannot be routinely used. 
Importance of Quality Control in MR-Based

Stereotactic Direct Targeting

Despite all imaging technologies, there are some limitations that physicists or physicians cannot control [26]. Strict quality control is necessary to ensure that applied technologies are at their optimum level of performance, and most quantitative quality controls are performed with phantoms [27, 28]. MRI-based stereotactic targeting precision is now confirmed to minimize the risk of complications $[15,29]$ and to be associated with a high level of accuracy [30] (statistical in-plane error of $0.6 \mathrm{~mm})$.

Furthermore, it is necessary to address potential human and technological sources of errors on a routine basis: stereotactic device handling; numerical data transmission; double checks of the coordinates set on the frame; verification of the straightness of the cannula; brain volume variations following dural opening [31, 32] in relationship and to be controlled by the anesthetic protocol; and field inhomogeneity artifacts [33].

The magnitude of distortions depends on multiple parameters: type of sequence; field strength; and field inhomogeneity. The risk of significant distortion has been shown to be very low at the center of the phantom. This is an important result for validating the MR-based direct targeting technique.

Errors of localization using a localizer box can be due to structural irregularities in the box itself or to defective fiducial markers caused by the presence of air bubbles [34]. The number of N-shaped rods and the way the coordinates of rods are determined (geometric center, pixel with the highest intensity value or center of mass) can also induce errors of localization [35].

Errors of distortion found at the periphery of images raise another issue arising from the fact that the landmarks of the localizer box are in the periphery of the image. Hence, evaluating the impact of distortion on the localizer box depiction and the consequences for target coordinate measurement appeared to be an important concern for validating this study. Clearly, errors due to distortion not only affect the apparent location of the target but also the calculation of coordinates. Even though 3-T scanners sometimes produce greater detail, the apparent benefit is nullified if the coordinates are skewed [36, 37].

In this study, we have demonstrated that stereotactic localization is not affected by distortion in the periphery of the field of view with a 1.5-T magnet, in contrast to using a 3 -T magnet.

\section{Co-Registration Computed Tomography and MRI}

The option of acquiring a 3-T image and then registering to a stereotactic computed tomography (CT) or 1.5-T MRI exists. Neumann et al. [36] recommended for stereotactic procedures, a co-registration with 3-T MRI and CT. However, they showed also that, without CT co-registration, they obtained a comparable accuracy by using $1.5-\mathrm{T}$ VIBE sequences. Furthermore, they did not recommend the application of 7-T MR images, even with CT fusion, because of the absence of a good algorithm for automatic distortion.

It is true that a CT co-registration cannot correct intrinsic image distortions. In addition, we can add an error due to the fusion itself. On the contrary, CT/MRI fusion has been validated for the postoperative assessment of electrodes in many studies [38-40]. In gamma knife radiosurgery, Nakagawa et al. [41] showed more distortion with stereotactic MRI than with a 3-T image co-registered to a stereotactic CT. Thus, whatever preoperative imaging system is chosen, a postoperative MRI is the only way to validate the process.

\section{Conclusion}

We evaluated the accuracy of the procedure. By using a phantom with the Leksell frame, we have confirmed that 3T-MRI is associated with a higher risk of distortion and lead misplacement. Our results highlight the feasibility of methods based on simple statistical modeling of errors (error maps). The clinical significance of a given accuracy value is linked to the size of the target and to the size of the leads. The submillimeter accuracy, when reached, makes the procedure reliable.

\section{Statement of Ethics}

No ethic committee approval is required for a phantom study.

\section{Conflict of Interest Statement}

The authors report no conflicts of interest concerning the materials or methods used in this study or the findings specified in this paper.

\section{Funding Sources}

There are no funding sources to declare. 


\section{Author Contributions}

G.P.: performed data analysis and wrote the manuscript. E.C.S.: analyzed the data and contributed to the writing of the manuscript.
N.M.D.: participated in MRI acquisition. L.C., F.C., and J.P.: participated in acquisition and writing of the manuscript. P.C.: conceptualized the research, participated in the analysis and data interpretation and final approval.

\section{References}

1 Coubes $\mathrm{P}$, Echenne B, Roubertie A, Vayssière $\mathrm{N}$, Tuffery S, Humbertclaude V, et al. [Treatment of early-onset generalized dystonia by chronic bilateral stimulation of the internal globus pallidus. Apropos of a case]. Neurochirurgie. 1999 May;45(2):139-44.

2 Coubes P, Roubertie A, Vayssiere N, Hemm S, Echenne B. Treatment of DYT1-generalised dystonia by stimulation of the internal globus pallidus. Lancet. 2000 Jun;355(9222): $2220-1$.

3 Coubes P, Cif L, El Fertit H, Hemm S, Vayssiere N, Serrat S, et al. Electrical stimulation of the globus pallidus internus in patients with primary generalized dystonia: long-term results. J Neurosurg. 2004 Aug;101(2):189-94.

4 Vidailhet M, Vercueil L, Houeto JL, Krystkowiak P, Benabid AL, Cornu P, et al.; French Stimulation du Pallidum Interne dans la Dystonie (SPIDY) Study Group. Bilateral deepbrain stimulation of the globus pallidus in primary generalized dystonia. N Engl J Med. 2005 Feb;352(5):459-67.

5 Amirnovin R, Williams ZM, Cosgrove GR, Eskandar EN. Experience with microelectrode guided subthalamic nucleus deep brain stimulation. Neurosurgery. 2006 Feb;58(1 Suppl):ONS96-102.

6 Hariz GM, Blomstedt P, Koskinen LO. Longterm effect of deep brain stimulation for essential tremor on activities of daily living and health-related quality of life. Acta Neurol Scand. 2008 Dec;118(6):387-94.

7 Gross RE, Krack P, Rodriguez-Oroz MC, Rezai AR, Benabid AL. Electrophysiological mapping for the implantation of deep brain stimulators for Parkinson's disease and tremor. Mov Disord. 2006 Jun;21(Suppl 14):S25983.

8 Starr PA, Christine CW, Theodosopoulos PV, Lindsey N, Byrd D, Mosley A, et al. Implantation of deep brain stimulators into the subthalamic nucleus: technical approach and magnetic resonance imaging-verified lead locations. J Neurosurg. 2002 Aug;97(2):370-87.

9 Coubes P, Vayssiere N, El Fertit H, Hemm S, Cif L, Kienlen J, et al. Deep brain stimulation for dystonia. Surgical technique. Stereotact Funct Neurosurg. 2002;78(3-4):183-91.

10 Willinek WA, Schild HH. Clinical advantages of 3.0 T MRI over 1.5 T. Eur J Radiol. 2008 Jan; 65(1):2-14.

11 Schmitz BL, Aschoff AJ, Hoffmann MH, Grön G. Advantages and pitfalls in 3T MR brain imaging: a pictorial review. AJNR Am J Neuroradiol. 2005 Oct;26(9):2229-37.
12 Boutet A, Hancu I, Saha U, Crawley A, Xu DS, Ranjan M, et al. 3-Tesla MRI of deep brain stimulation patients: safety assessment of coils and pulse sequences. J Neurosurg. 2019 Feb;132(2):586-94.

13 Voormolen EH, Diederen SJ, Woerdeman P, van der Sprenkel JW, Noordmans HJ, Visser F, et al. Implications of Extracranial Distortion in Ultra-High-Field Magnetic Resonance Imaging for Image-Guided Cranial Neurosurgery. World Neurosurg. 2019 Jun; 126:e250-8

14 Juhász A, Kovács N, Perlaki G, Büki A, Komoly S, Kövér F, et al. A subthalamicus mag célkoordinátáinak összehasonlítása 1 és 3 Tesla MR-vizsgálattal mély agyi stimulációs műtétek tervezése során. Ideggyogy Sz. 2018 Nov;71(11-12):405-10.

15 Maldonado IL, Roujeau T, Cif L, Gonzalez V, El-Fertit H, Vasques X, et al. Magnetic resonance-based deep brain stimulation technique: a series of 478 consecutive implanted electrodes with no perioperative intracerebral hemorrhage. Neurosurgery. 2009 Dec;65(6 Suppl):196-201.

16 Zrinzo L. Pitfalls in precision stereotactic surgery. Surg Neurol Int. 2012;3(2 Suppl 1):S5361.

17 Lumsden DE, Ashmore J, Charles-Edwards G, Lin JP, Ashkan K, Selway R. Accuracy of stimulating electrode placement in paediatric pallidal deep brain stimulation for primary and secondary dystonia. Acta Neurochir (Wien). 2013 May;155(5):823-36.

18 Maciunas RJ, Galloway RL Jr, Latimer JW. The application accuracy of stereotactic frames. Neurosurgery. 1994 Oct;35(4):68294.

19 Shamir RR, Joskowicz L, Spektor S, Shoshan $\mathrm{Y}$. Target and trajectory clinical application accuracy in neuronavigation. Neurosurgery. 2011 Mar;68(1 Suppl Operative):95-101; discussion 101-2.

20 Cho ZH, Min HK, Oh SH, Han JY, Park CW, Chi JG, et al. Direct visualization of deep brain stimulation targets in Parkinson disease with the use of 7-tesla magnetic resonance imaging. J Neurosurg. 2010 Sep;113(3):639-47.

21 Kerl HU, Gerigk L, Pechlivanis I, Al-Zghloul M, Groden C, Nölte IS. The subthalamic nucleus at 7.0 Tesla: evaluation of sequence and orientation for deep-brain stimulation. Acta Neurochir (Wien). 2012 Nov;154(11):205162.

22 Slavin KV, Thulborn KR, Wess C, Nersesyan $H$. Direct visualization of the human subthalamic nucleus with 3T MR imaging. AJNR Am J Neuroradiol. 2006 Jan;27(1):80-4.
23 Vayssiere N, Hemm S, Cif L, Picot MC, Diakonova N, El Fertit H, et al. Comparison of atlas- and magnetic resonance imaging-based stereotactic targeting of the globus pallidus internus in the performance of deep brain stimulation for treatment of dystonia. J Neurosurg. 2002 Apr;96(4):673-9.

24 Bernstein MA, Huston J 3rd, Ward HA. Imaging artifacts at 3.0T. J Magn Reson Imaging. 2006 Oct;24(4):735-46.

25 Tavares WM, Tustumi F, da Costa Leite C, Gamarra LF, Amaro E Jr, Teixeira MJ, et al. An image correction protocol to reduce distortion for 3-T stereotactic MRI. Neurosurgery. 2014 Jan;74(1):121-6.

26 Georgi JC, Stippich C, Tronnier VM, Heiland $\mathrm{S}$. Active deep brain stimulation during MRI: a feasibility study. Magn Reson Med. 2004 Feb;51(2):380-8.

27 Lauzon CB, Caffo BC, Landman BA. Towards Automatic Quantitative Quality Control for MRI. Proc SPIE Int Soc Opt Eng. 2012 Feb; 8314:910819.

28 Wang D, Doddrell DM. A proposed scheme for comprehensive characterization of the measured geometric distortion in magnetic resonance imaging using a three-dimensional phantom. Med Phys. 2004 Aug;31(8):2212-8.

29 Foltynie T, Zrinzo L, Martinez-Torres I, Tripoliti E, Petersen E, Holl E, et al. MRI-guided STN DBS in Parkinson's disease without microelectrode recording: efficacy and safety. J Neurol Neurosurg Psychiatry. 2011 Apr; 82(4):358-63.

30 Vayssiere N, Hemm S, Zanca M, Picot MC Bonafe A, Cif L, et al. Magnetic resonance imaging stereotactic target localization for deep brain stimulation in dystonic children. J Neurosurg. 2000 Nov;93(5):784-90.

31 Khan MF, Mewes K, Gross RE, Skrinjar O Assessment of brain shift related to deep brain stimulation surgery. Stereotact Funct Neurosurg. 2008;86(1):44-53.

32 Pollo C, Vingerhoets F, Pralong E, Ghika J, Maeder P, Meuli R, et al. Localization of electrodes in the subthalamic nucleus on magnetic resonance imaging. J Neurosurg. 2007 Jan; 106(1):36-44.

33 Bourgeois G, Magnin M, Morel A, Sartoretti S, Huisman T, Tuncdogan E, et al. Accuracy of MRI-guided stereotactic thalamic functional neurosurgery. Neuroradiology. 1999 Sep;41(9):636-45.

34 Park JH, Han JH, Kim CY, Oh CW, Kim DG, Suh TS, et al. Effect of fiducial marker defects on stereotactic target localization in the Leksell stereotactic system. Med Biol Eng Comput. 2011 Jul;49(7):775-82. 
35 Lemieux L, Jagoe R. Effect of fiducial marker localization on stereotactic target coordinate calculation in CT slices and radiographs. Phys Med Biol. 1994 Nov;39(11):1915-28.

36 Neumann JO, Giese H, Biller A, Nagel AM, Kiening K. Spatial Distortion in MRI-Guided Stereotactic Procedures: Evaluation in 1.5-, 3 and 7-Tesla MRI Scanners. Stereotact Funct Neurosurg. 2015;93(6):380-6.

37 Schmidt MA, Wells EJ, Davison K, Riddell AM, Welsh L, Saran F. Stereotactic radiosurgery planning of vestibular schwannomas: is MRI at 3 Tesla geometrically accurate? Med Phys. 2017 Feb;44(2):375-81.
38 Geevarghese R, O'Gorman Tuura R, Lumsden DE, Samuel M, Ashkan K. Registration Accuracy of CT/MRI Fusion for Localisation of Deep Brain Stimulation Electrode Position: An Imaging Study and Systematic Review. Stereotact Funct Neurosurg. 2016;94(3):15963.

39 Mirzadeh Z, Chapple K, Lambert M, Dhall R, Ponce FA. Validation of CT-MRI fusion for intraoperative assessment of stereotactic accuracy in DBS surgery. Mov Disord. 2014 Dec;29(14):1788-95.
40 O'Gorman RL, Jarosz JM, Samuel M, Clough C, Selway RP, Ashkan K. CT/MR image fusion in the postoperative assessment of electrodes implanted for deep brain stimulation. Stereotact Funct Neurosurg. 2009;87(4):20510.

41 Nakagawa H, Ninomiya T, Yamashita T, Takada M. Treatment with the Neutralizing Antibody against Repulsive Guidance Molecule-a Promotes Recovery from Impaired Manual Dexterity in a Primate Model of Spinal Cord Injury. Cereb Cortex. 2019 Feb; 29(2):561-72. 